Classification

Physics Abstracts

$62.60-78.35$

\title{
BRILLOUIN SCATTERING FROM SUPERCOOLED WATER
}

\author{
J. TEIXEIRA and J. LEBLOND \\ Laboratoire de Résonance Magnétique (*) \\ Ecole de Physique et Chimie, 10, rue Vauquelin 75231 Paris Cedex 05, France
}

(Reçu le 19 décembre 1977, accepté le 21 février 1978)

\begin{abstract}
Résumé. - Nous présentons quelques résultats expérimentaux originaux pour la vitesse et absorption sonore dans l'eau surfondue dans le domaine de température qui s'étend jusqu'à $-20^{\circ} \mathrm{C}$. Ces résultats montrent une rapide variation avec la température et peuvent être analysés avec des équations analogues à celles qui décrivent les phénomènes critiques.
\end{abstract}

\begin{abstract}
We present new experimental results for sound velocity and sound absorption of supercooled water in the temperature range down to $-20^{\circ} \mathrm{C}$. The results show a rapid variation with temperature that can be analysed according to equations analogous to the critical phenomena laws.
\end{abstract}

1. Introduction. - Supercooled water has recently aroused considerable interest because of its anomalous behaviour in the metastable region.

Some physical properties such as density, heat capacity, isothermal compressibility, etc... show a rapid variation as the temperature of water decreases [1].

The explanation of this behaviour is not clear and different interpretations have been proposed either in terms of a lambda-transition or in terms of a mechanical instability.

The first possibility, proposed by Angell et al. [2] associates the thermodynamic singularity to a cooperative formation of an open hydrogen-bonded network. If this explanation is correct, one may observe at some temperature near $-45^{\circ} \mathrm{C}$ a rapid decrease of the heat capacity, $C_{\mathrm{p}}$, with decreasing temperature, in order to meet at $150 \mathrm{~K}$ the heat capacity of vitreous water without exhaustion of all the entropy of fusion. Nevertheless, the results obtained by Sugisaki et al. [3] for the heat capacity of vitreous ice indicate [4] that there is no continuity of state between supercooled water and vitreous ice.

The second one does not require any continuity of the curve $C_{\mathrm{p}}(T)[1,5]$ and is supported by the close correspondance between the temperature of the thermodynamic singularity $\left(T_{\mathrm{s}} \simeq-45^{\circ} \mathrm{C}\right)$ and the experimental value of the homogeneous nucleation temperature [6].

On the other hand, the strong dependence of vis-

(*) E.R.A. 365 du C.N.R.S. cosity on temperature [7] could also be explained in terms of a diffusive soft mode $[8,9]$.

Anyway, from an experimental point of view, we can describe this anomalous behaviour rather well with some characteristic critical exponents as in critical phenomena. This procedure has been used for many properties and leads to numerical results in good agreement with experiment if the critical temperature $T_{\mathrm{s}}$ is set to be $228 \mathrm{~K}$ [1].

Brillouin light scattering was shown to be a valuable method in obtaining experimental information on dynamical properties of supercooled water. Previous results were obtained for $T>-10^{\circ} \mathrm{C}$ in large volume sample [10] and were found to be in good agreement with critical laws [11]. On the other hand using the capillary method, we have been able to supercool water down to $-20^{\circ} \mathrm{C}$ and the aim of this letter is to report new results on the propagation of a high frequency sound wave.

2. Experimental set-up and results. - We have used the glass capillary method to extend the experimental range of supercooled water. In fact, because of homogeneous nucleation, the minimum freezing temperature is a linear function of the logarithm of the volume of the sample [6]. Thermal equilibrium was obtained by regulation of an ethanol bath in which the capillary was placed in a set-up analogous to that used by Hallett [7]. The temperature stability was better than $0.1^{\circ} \mathrm{C}$ and the temperature measurement accuracy was better than $0.2^{\circ} \mathrm{C}$.

Light from a single frequency $\mathrm{Ar}^{+}$laser was focused 
in the capillary. The light scattered at $90^{\circ}$ is dispersed by a linear pressure scanned Fabry-Perot interferometer with a free spectral range equal to $14.77 \mathrm{GHz}$. The overall finesse of the spectrometer was greater than 50. The detector was a phototube connected to a photon counter and a digital-analogue converter. The parasitic light arising from the wall of the capillary makes intensity analysis impossible. However it does not influence the position of the Brillouin peaks.

The sound velocity, $v$, and the sound absorption, $\alpha / f^{2}$, have been calculated according to the following formulae :

$$
\begin{gathered}
v=\frac{\omega_{\mathrm{B}} \lambda_{0}}{4 \pi n} \cdot \frac{1}{\sin \theta / 2} \\
\frac{\alpha}{f^{2}}=\frac{2 \pi^{2} \Gamma_{\mathrm{B}}}{C \omega_{\mathrm{B}}^{2}}
\end{gathered}
$$

where $\lambda_{0}=0.5145 \mu \mathrm{m}$ is the wavelength of the incident light, $n$ the refractive index of the water, $\omega_{\mathbf{B}}$ the Brillouin shift, $\theta=90^{\circ}$ the scattering angle, $\alpha$ the absorption coefficient, $f=\omega_{\mathrm{B}} / 2 \pi$ the sound wave frequency, $C \simeq v$ the low frequency sound velocity and $\Gamma_{\mathbf{B}}$ the Brillouin line width. Typically (at $0{ }^{\circ} \mathrm{C}$ ) $\omega_{\mathrm{B}} / 2 \pi=5.11 \mathrm{GHz}$ and $\Gamma_{\mathrm{B}} / 2 \pi \simeq 505 \mathrm{MHz}$.

The finesse of the spectrometer corresponds to a width of the instrumental profile approximately equal to $300 \mathrm{MHz}$ which is enough for a good separation of the Brillouin peaks from the central peak.

The experimental results obtained for $v$ and $\alpha / f^{2}$ are plotted in figures 1 and 2 against temperature. The experimental absolute errors are about $5 \mathrm{~m} . \mathrm{s}^{-1}$ and $10^{-14} \mathrm{~s}^{2} \cdot \mathrm{m}^{-1}$ respectively.

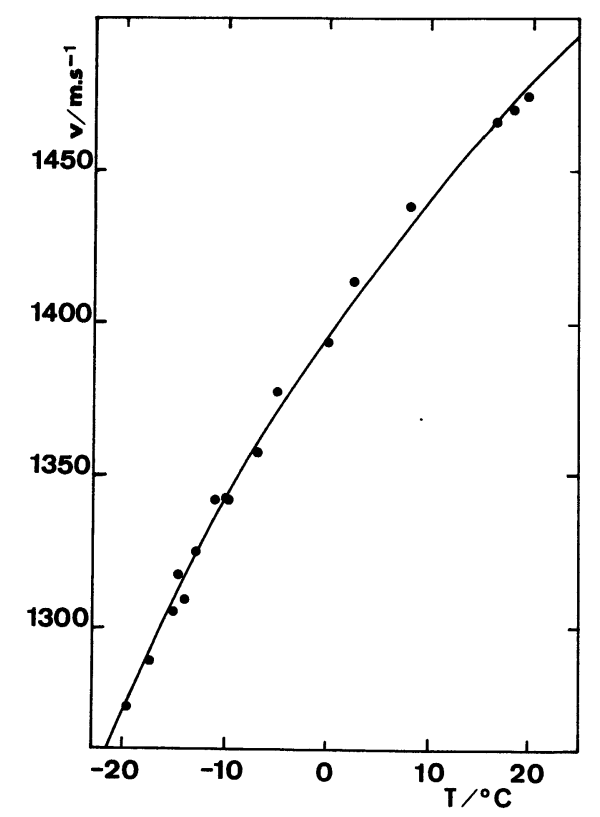

FIG. 1. - Plot of the experimental values of the sound velocity in water against temperature. The solid line represents the best fit which can be made with a critical law.

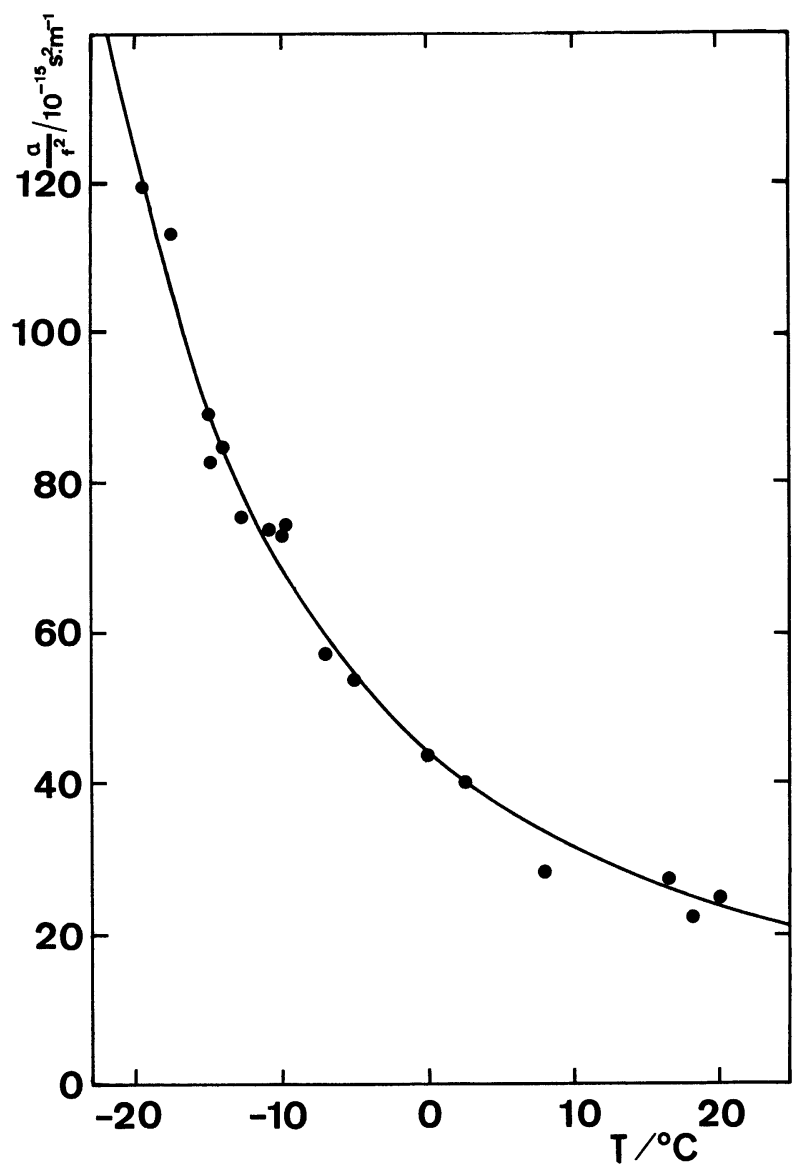

Fig. 2. - Plot of the sound absorption against temperature. The solid line represents the best fit which can be made with a critical law.

We see that $v$ and $\alpha / f^{2}$ have a rapid variation with temperature which becomes larger at lower temperatures.

3. Discussion. - In order to test the validity of a law like

$$
A=A_{0} \varepsilon^{x}
$$

for each property $A$, where $\varepsilon \equiv\left(T-T_{\mathrm{s}}\right) / T_{\mathrm{s}}, A_{0}$ is a constant and $x$ a critical exponent, a least-square method has been used to fit the experimental results. We have suppressed the points above $10^{\circ} \mathrm{C}$ in this analysis to avoid the spurious effects of the variation at high temperature.

We obtain :

$$
\begin{gathered}
v=1798.3 \varepsilon^{0.156} \mathrm{~m} \cdot \mathrm{s}^{-1} \\
\frac{\alpha}{f^{2}}=267.2 \times 10^{-17} \varepsilon^{-1.73} \mathrm{~s}^{2} \cdot \mathrm{m}^{-1}
\end{gathered}
$$

this is in good agreement with the results of Rouch et al. [11], i.e., $v=1782.4 \varepsilon^{0.1523} \mathrm{~m} \cdot \mathrm{s}^{-1}$ and $\alpha / f^{2}=244.84 \varepsilon^{-1.7882} \mathrm{~s}^{2} \cdot \mathrm{m}^{-1}$.

The relative errors were about $0.5 \%$ and $7.5 \%$ respectively. 
The thermodynamic form of the sound velocity, $v$, is given by

$$
\frac{1}{v^{2}}=\rho\left[k_{T}-\frac{T V \alpha_{\mathrm{p}}^{2}}{C_{\mathrm{p}}}\right]
$$

where $\rho$ is the mass density, $k_{T}$ the isothermal compressibility, $V$ the volume and $\alpha_{\mathrm{p}}$ the thermal expansion.

The numerical fit done by Speedy and Angell [1], with laws such as formula (1) for the experimental values of $k_{T}$ and $\rho$ gives exponents of -0.349 and 0.0243 respectively. Consequently $\rho k_{T}$ must change as $\varepsilon^{-0.325}$. Because we find a thermal dependence for the sound velocity as $\varepsilon^{0.156}$, which corresponds to a variation of $1 / v^{2}$ with temperature like $\varepsilon^{-0.312}$, we conclude that the essential dependence on temperature is explained by the first term of Eq. (6).

On the other hand, the equation for the sound attenuation is given by :

$$
\frac{\alpha}{f^{2}}=\frac{2 \pi^{2}}{\rho v^{3}}\left(\frac{4}{3} \eta_{\mathrm{s}}+\eta_{\mathrm{B}}+\frac{\gamma-1}{C_{\mathrm{p}}} \lambda\right)
$$

where $\eta_{\mathrm{s}}$ and $\eta_{\mathrm{B}}$ are the shear and bulk viscosities respectively, $\gamma=C_{\mathrm{p}} / C_{\mathrm{v}}$ and $\lambda$ the thermal conductivity. This expression gives the actual behaviour of $\alpha / f^{2}$ if we retain, near $T_{\mathrm{s}}$, only the two first terms. In fact the ratio $\eta_{\mathrm{B}} / \eta_{\mathrm{s}}$ can be deduced from the results of Hallett [7] and our experimental results for $\alpha / f^{2}$ using Eq. (7) and neglecting the last terms $(\gamma-1 \simeq 0)$. Because we find in all the analysed temperature range a ratio $\eta_{\mathrm{B}} / \eta_{\mathrm{s}}$ which is about 2 , we retain the same thermal dependence for the two viscosities.

Under these conditions, we obtain

$$
\frac{\alpha}{f^{2}} \sim \frac{\eta_{\mathrm{s}}+\eta_{\mathrm{B}}}{v^{3}} \sim \varepsilon^{-1.476} \varepsilon^{-0.468}=\varepsilon^{-1.944}
$$

where we have used for $\eta_{\mathrm{s}}$ the results of Hallett [7]. This result is in a good agreement with our experimental one (Eq. (5)).

The anomalous behaviour of sound velocity and sound absorption are thus confirmed. Our results support the existence of a thermodynamic singularity near $228 \mathrm{~K}$. More experimental work is of course necessary to explain the reasons of this peculiar behaviour. In particular it would seem necessary to approach the critical temperature $T_{\mathrm{s}}$, and to try to extend our temperature range to the vicinity of $T_{\mathrm{s}}$.

Acknowledgments. - The authors are grateful to Dr Rouch for helpful discussions.

\section{References}

[1] Speedy, R. J. and ANGell, C. A., J. Chem. Phys. 65 (1976) 851.

[2] Angell, C. A., Shuppert, J., Tucker, J. C., J. Phys. Chem. 77 (1973) 3092

[3] Sugisaki, M., Suga, H. and Seki, M., Bull. Chem. Soc. Jpn. 41 (1968) 2591.

[4] Johari, G. P., Philos. Mag. 35 (1977) 1077.

[5] Rasmussen, D. H. and MaC Kenzie, A. P., J. Chem. Phys. 59 (1973) 5003.

[6] SkriPov, V. P., Metastable Liquids (John Wiley, New York) 1974 , p. 138.
[7] Hallett, J., Proc. Phys. Soc. London 82 (1963) 1046.

[8] Schneider, T., Brout, R., Thomas, H. and Feder, J., Phys. Rev. Lett. 25 (1970) 1423.

[9] Haus, J. W., MeiJer, P. H. E., Phys. Rev. 14 (1976) 2285.

[10] Rouch, J., LAI, C. C. and Chen, S. H., J. Chem. Phys. 65 (1976) 4016.

[11] Rouch, J., LAI, C. C. and Chen, S. H., J. Chem. Phys. 66 (1977) 5031. 